\title{
Ontologia como Representação do Conhecimento: aplicação no curso de Formação Continuada em Tecnologias Educacionais na Web
}

Andreza Regina Lopes da Silva - Universidade Federal de Santa Catarina/Pósgraduação em Engenharia e Gestão do Conhecimento - andrezalopes.ead@gmail.com

Michele Andréia Borges - Universidade Federal de Santa Catarina/Pós-graduação em Engenharia e Gestão do Conhecimento - micheleandborges@gmail.com

Maria Cristina Pfeiffer Fernandes - Centro de Ciências e Ensino Superior a Distância do Estado do Rio de Janeiro (CECIERJ) - pfeiffer@cederj.rj.gov.br

Viviane Sartori - Universidade Federal de Santa Catarina /Pós-graduação em Engenharia e Gestão do Conhecimento - vivi.sartori19@gmail.com

Fernando José Spanhol - Universidade Federal de Santa Catarina /Pós-graduação em Engenharia e Gestão do Conhecimento - profspanhol@gmail.com

Resumo. Programas de educação a distância, disponibilizados em ambientes virtuais de aprendizagem, possuem um espaço de criação e compartilhamento de conhecimentos, que precisam ser devidamente gerenciados para possibilitar seu reuso. A ontologia apresenta-se como um recurso que proporciona a explicitação do conhecimento consensual e compartilhado, numa linguagem formal, de um dado domínio. Nesta pesquisa, tem-se por objetivo, apresentar uma ontologia para armazenamento estruturado da informação e do conhecimento produzido num curso, na modalidade a distância. Um resultado importante, alcançado, foi a constatação de que a representação do conhecimento, através de ontologias, é um recurso potencial para apoio à tomada de decisão da equipe gestora, num ambiente virtual de aprendizagem, que oferece cursos on-line.

Palavras-chaves: Educação a Distância. Ontologias. Cursos on-line. Conhecimento.

\section{Ontology as Knowledge Representation: Application in the course of Continuing Education in Educational Technologies Web}

\begin{abstract}
Distance education programs, available in virtual learning environments, have a space of creation and sharing of knowledge that need to be properly managed in order to enable its reuse. Ontology is presented as a resource that provides explicit knowledge, shared and consensual, in a formal language, a given domain. This research has as objective to present ontology for structured storage of information and knowledge produced in a course, in distance mode. An important result, achieved, was finding that knowledge representation through ontologies is a potential resource to support decision-making for the management team, in a virtual learning environment, which offers online courses.
\end{abstract}

Keywords: Distance Learning. Ontology. Online Course. Knowledge. 


\section{Introdução}

O uso das tecnologias digitais, atrelado a Educação a Distância (EaD), tem ampliado a possibilidade de educação para a sociedade. No entanto, para que ocorra a aprendizagem nesta modalidade de ensino é necessário conjugar recursos em diferentes mídias, por exemplo, material impresso, rádio, televisão, computador e mais recentemente a Internet, como artefato mediador na disseminação e compartilhamento do conhecimento.

Da integração entre os diferentes e significativos papeis representativos que a EaD vem apresentando junto à sociedade do conhecimento, a Diretoria dos Cursos de Extensão da Fundação Centro de Ciências e Ensino Superior a Distância do Estado do Rio de Janeiro (CECIERJ) oferece cursos de extensão em diferentes áreas do conhecimento, dentre as quais, a área de Tecnologia Educacional. Para o estudo de caso apresentado neste artigo, foi selecionado um curso desta área, Formação Continuada em Tecnologias Educacionais na Web (FCTEWeb), oferecido semestralmente em dois Módulos, 1 e 2, cujo objetivo é usar a filosofia "fazer-aprendendo" para trabalhar com os professores cursistas algumas aplicações gratuitas da Web 2.0, como uma alternativa educacional para sua prática docente.

Dentro deste cenário, a pesquisa caracteriza-se como científica, interdisciplinar, exploratória e com abordagem qualitativa. Tem como objetivo, desenvolver uma ontologia para o curso on-line FCTEWeb, utilizando-se para a coleta de dados, a busca bibliográfica, bem como o estudo de caso pautado na observação participante. O artigo está dividido em 6 (seis) seções, valendo destacar a metodologia onde procurou-se descrever o escopo do estudo de caso, a ferramenta utilizada e as etapas de construção da ontologia associada ao curso on-line FCTEWeb. Destaca-se ainda, o mapeamento, armazenamento estruturado da informação e do conhecimento produzido pelos participantes durante um curso on-line, pode auxiliar a tomada de decisão dos professores coordenadores e gestores iniciantes ou já iniciados na $\mathrm{EaD}$, bem como, contribuir com o avanço científico da área.

\section{Ontologia}

O termo ontologia não é novo. Há muito tempo, filósofos como Aristóteles, empregam esse termo na tentativa de classificar e descrever o conhecimento no mundo.

A Inteligência Artificial (IA), surgida em 1950, adotou o termo ontologia para descrever o que pode ser representado de um determinado domínio em um programa computacional. Dentro de um domínio de conhecimento, a ontologia consiste numa estrutura formal de conceitos e relações entre estes, além de um conjunto de axiomas que restringe a interpretação desta estrutura e permite a derivação de conhecimento novo a partir do conhecimento factual (GUIZZARDI, 2005).

As ontologias têm contribuído para facilitar a comunicação e o processamento de informação semântica, tanto entre humanos, quanto entre sistemas computacionais. São comumente utilizadas para promover a interoperabilidade entre sistemas, ao representarem os dados compartilhados por diversas aplicações (UCHOLD; GRÜNINGER, 2004). Ampliando a discussão, Berners-Lee, Hendler e Lassila (2001) destacam, também, as ontologias como artefatos fundamentais para a Web Semântica além de meta dados que juntos conseguem estruturar e dar significado ao conteúdo das páginas web.

A razão pelas quais ontologias têm apresentado relevância em diferentes cenários pode ser atribuída à sua própria definição e o que ela assegura: uma 
especificação explícita de uma conceitualização (GRUBER, 1993). Borst (1997) aponta que a ontologia é uma especificação explícita e formal de uma conceitualização compartilhada. Freitas (2003) elucida os requisitos dessa definição entendendo como especificação explícita as definições de conceitos, instâncias, relações, restrições e axiomas; por formal, definição declarativamente definida, portanto, compreensível para agentes e sistemas; conceitualização são as definições que se trata de um modelo abstrato de uma área de conhecimento ou de um universo limitado de discurso; compartilhada são definições de um conhecimento consensual.

Tratando-se do aspecto da especificação explícita Gruber (1996), Noy e Guinness (2001) detalham os componentes básicos de uma ontologia que determinam as conceitualizações ou modelos abstratos de fenômenos do mundo real como:

- Classes: também chamadas comumente de conceitos, podem ser do tipo abstrato ou concreto, simples ou composto, reais ou fictícios. Um conceito pode ser "qualquer coisa" a respeito de "algo" que estamos explicando, e por esse motivo pode ser a descrição de uma tarefa, função, ação, estratégia ou um processo de raciocínio. As classes são organizadas em taxonomia que, por sua vez, são empregados para organizar o conhecimento ontológico usando generalização e especialização através do qual podem ser aplicadas relações de herança simples e múltipla, entre as classes. Além disso, as classes possuem atributos dos quais revelam propriedades importantes do conceito.

- Relações e funções: relações é um tipo de interação entre os conceitos de um domínio e seus atributos. Já as funções são um tipo especial de relação (p. e., Exponencial (x), Media Final (P1, P2)).

- Axiomas: utilizados para modelar sentenças sempre verdadeiras. Os axiomas podem ser utilizados para vários fins, tais como: impor restrições, verificar a correção e realizar dedução de novas informações. Axiomas são usados para restringir a interpretação e uso dos conceitos envolvidos na ontologia.

- Instâncias: as instâncias ou indivíduos de uma ontologia representam elementos do domínio associados a um conceito específico, ou seja, os próprios dados. As instâncias possuem atributos que são propriedades relevantes que descrevem as instâncias de um conceito.

Além disso, as ontologias podem ser divididas em dois grupos específicos: "ontologias leves" (lightweight ontologies) e “ontologias pesadas" (heavyweight ontologies). As ontologias leves incluem conceitos, relações e instâncias. Já as ontologias pesadas contemplam todos os aspectos de uma ontologia leve acrescentandose axiomas e restrições (CORCHO; FERNÁNDEZ-LÓPES; GÓMEZ-PEREZ, 2003).

Além dessas características básicas, Guarino (1997) classificou as ontologias em quatro tipos:

- Ontologias de alto nível: descrevem conceitos bastante gerais como espaço, tempo, matéria, objeto, ações, eventos, etc., independentes de um problema particular ou domínio.

- Ontologias de domínio: descrevem o vocabulário relacionado a um domínio genérico (por exemplo, medicina, educação a distância, etc.).

- Ontologias de tarefa: descrevem o vocabulário relacionado a uma atividade ou tarefa (por exemplo, diagnóstico ou vendas).

- Ontologias de aplicação: descrevem conceitos em função tanto de um domínio particular quanto de uma tarefa, que muitas vezes são especializações de ambas as ontologias relacionadas. 
O tipo em que uma aplicação de ontologia estará caracterizada vai, depender do escopo e objetivo a que essa se propõe. Aqui nesta pesquisa a discussão esta à luz da prática da EaD num curso on-line de formação continuada via web e é neste sentido que a seção seguinte foi elaborada.

\section{Educação a distância e formação continuada via web}

A EaD tem conquistado espaço para discussão científica, prática educacional e fomento junto à diferentes órgãos institucionais, já que se configura como uma prática educativa de cunho social, cada vez mais pertencente ao mundo atual, em função da possibilidade de associá-la com as novas tecnologias, permitindo uma educação virtual e a distância. Entende-se então, que EaD é uma modalidade educacional de cunho social no qual se estuda e ensina a distância, mas não se está distante do aluno. Neste sentido, Moore e Kearsley (2007) conceituam a educação a distância como espaço de aprendizado planejado que exige técnicas especiais de criação do curso, comunicação por meio de várias tecnologias e disposições organizacionais e administrativas especiais. $\mathrm{Na} \mathrm{EaD,} \mathrm{professores} \mathrm{e} \mathrm{alunos,} \mathrm{geralmente} \mathrm{não} \mathrm{estão} \mathrm{juntos,} \mathrm{estão} \mathrm{separados}$ física e/ou temporalmente, entretanto, podem estar conectados pelas tecnologias digitais. As potencialidades desta modalidade educacional são vastas entre os seus diferentes atores envolvidos.

Entretanto, o caminho da EaD é complexo, ou seja, se por um lado existem elementos que potencializam seu desenvolvimento, por outro, existem aqueles que limitam o seu sucesso. Devido ao crescimento e facilidade de acesso à Web atualmente, pode-se prever o cenário futuro com expectativas positivas, com o avanço dessa modalidade educacional proporcionando assim, uma grande oferta educacional para um número cada vez maior de pessoas que, sem este recurso, teriam maiores dificuldades de ingresso em cursos de formação e/ou aperfeiçoamento.

\section{Metodologia e ferramenta de construção da ontologia}

Metodologias têm sido desenvolvidas no intuito de sistematizar a construção e a manipulação de ontologias (FERNANDÉZ-LOPEZ, 1999). Para a construção da ontologia do curso FCTEWeb foi utilizada a ferramenta OntoKEM e a ferramenta Protégé. No processo de desenvolvimento de ontologias, usualmente, são aceitas as atividades de especificação, conceitualização, formalização, implementação e manutenção, com suas respectivas tarefas, que podem não estar totalmente contempladas em uma única metodologia para desenvolvimento de uma ontologia.

A ferramenta OntoKEM baseia-se nos pressupostos de três metodologias de construção de ontologias, como mostra a Tabela 1, com o intuito de combiná-las para se obter um melhor resultado nas atividades de especificação, conceitualização, formalização e documentação de ontologias (RAUTENBERG et al., 2010).

Tabela 1 - Metodologias utilizadas no OntoKEM.

\begin{tabular}{|c|l|}
\hline Metodologia & \multicolumn{1}{|c|}{ Contribuição } \\
\hline $\begin{array}{c}\text { Ontology Development 101 (Noy; } \\
\text { Mcguiness, 2008) }\end{array}$ & Processo iterativo para o desenvolvimento de ontologias. \\
\hline $\begin{array}{c}\text { On-to_knowledge } \\
\text { (Studer et al., 2003) }\end{array}$ & $\begin{array}{l}\text { Definição do domínio e escopo da ontologia. E utilização de } \\
\text { questões de competência. }\end{array}$ \\
\hline $\begin{array}{c}\text { Methontology } \\
\text { (Gômez-Pérez, 2004) }\end{array}$ & Conjunto de artefatos de documentação. \\
\hline
\end{tabular}

Fonte: Adaptado de Rautenberg et al., (2010). 
A utilização da ferramenta Protégé foi motivada por uma gama de fatos, como: plataforma livre de código-aberto que provê um conjunto de ferramentas para construir modelos de domínio e aplicações baseadas em conhecimento com ontologias. O Protégé é também um poderoso editor de ontologias para as novas linguagens desenvolvidas em função da Web Semântica. Uma das grandes vantagens do Protegé-2000, é que o usuário não precisa se preocupar com a sintaxe da linguagem usada na Web, bastando se concentrar nos conceitos e nas relações do domínio e nos fatos que precisam ser representados (GOÑI, FERNANDES, LUCENA, 2002; PROTÉGÉ, 2001). Outras vantagens são: a quantidade significativa de plug-ins que potencializam a sua funcionalidade; o mecanismo de inferência para a verificação de ontologias e a classificação automática (MOLOSSI, 2008). Além disso, a ontologia desenvolvida para o curso FCTEWeb está delimitada no grupo das ontologias leves, ou seja, foram atribuídos conceitos, taxonomias, relações e propriedades que descrevem os conceitos do curso.

Segundo Almeida e Bax (2003), as ontologias podem ser classificadas, também, quanto ao seu tipo. Considerando o tipo da ontologia em relação ao grau de formalismo, a ontologia do curso FCTEWeb pode ser classificada como semi-informal, isto é, expressa-se em linguagem natural de forma restrita e estruturada. Em relação ao conteúdo, pode ser classificada como uma ontologia de aplicação que contém as definições necessárias para modelar o conhecimento da aplicação em questão.

\section{Desenvolvimento da ontologia do curso FCTEWeb}

O desenvolvimento da ontologia do curso FCTWeb obedeceu à definição especificada no conceito de uma ontologia leve e a utilização da metodologia da ferramenta OntoKEM. Para tanto, seguiu-se as etapas de definição do escopo da ontologia, definição das perguntas de competências, extração dos vocábulos a partir das perguntas de competência, definição das relações possíveis, também, extraídas das perguntas de competência e, por fim, a hierarquização das classes geradas em função das etapas anteriores.

O escopo desta pesquisa está circunscrito ao curso on-line de Formação Continuada em Tecnologias Educacionais na Web (FCTEWeb), oferecido pela Diretoria dos Cursos de Extensão da Fundação Centro de Ciências e Ensino Superior a Distância do Estado do Rio de Janeiro(CECIERJ). Este curso foi planejado no segundo semestre de 2009, por uma equipe multidisciplinar, formada por uma professora conteudista com especialização em informática educativa, um designer gráfico, um designer instrucional e um administrador do AVA (plataforma Moodle).

O curso FCTEWeb teve por objetivo refletir e discutir sobre a forma mais interativa de usar a Web 2.0. Além disso, através da filosofia "fazer-aprendendo", procurou-se trabalhar com os professores cursistas algumas aplicações gratuitas Web 2.0 de modo a refletir o seu uso como uma alternativa educacional para sua prática docente. É um curso gratuito oferecido, semestralmente em dois Módulos, 1 e 2, a professores em atividade, tendo prioridade aqueles que estão lecionando nas escolas públicas. Só poderão cursar o Módulo 2 os professores cursistas que forem aprovados no Módulo 1. Cada módulo tem carga horária de 30 horas e é oferecida certificação após sua conclusão.

Dentro deste cenário real de estudo, o objetivo em desenvolver uma ontologia do FCTEWeb, é o armazenamento estruturado da informação e do conhecimento 
produzido pelos participantes ao longo do curso, para apoiar a tomada de decisão da equipe gestora.

\subsection{Perguntas de competência, termos e relações}

As perguntas de competência elaboradas na ferramenta OntoKEM, foram baseadas no entendimento do domínio em estudo visando atender o objetivo proposto. Assim, foram desenvolvidas doze perguntas de competência, de forma aleatória, isto é, não se adotou uma sistematização. Ao criar as perguntas de competência a ferramenta OntoKEM subsidia a identificação de termos e relações, como mostra a Tabela 2.

Tabela 2 - Perguntas de competência, termos e relações.

\begin{tabular}{|c|c|c|}
\hline Perguntas de Competência & Termos Sugeridos & Relações Sugeridas \\
\hline Que atores formam a equipe do curso? & $\begin{array}{l}\text { Equipe } \\
\text { Aluno }\end{array}$ & $\begin{array}{l}\text { tem equipe } \\
\text { tem aluno }\end{array}$ \\
\hline $\begin{array}{l}\text { Qual o Ambiente Virtual de Aprendizagem } \\
\text { (AVA) que o curso utiliza? }\end{array}$ & $\begin{array}{c}\text { AVA } \\
\text { Moodle }\end{array}$ & $\begin{array}{l}\text { tem AVA } \\
\text { tem moodle }\end{array}$ \\
\hline O curso possui material didático? & Material Didático & tem material didático \\
\hline Quais os módulos do curso? & Módulo & tem módulo \\
\hline Os módulos possuem etapas? & Etapa & tem etapa \\
\hline O curso possui avaliação? & Avaliação & tem avaliação \\
\hline Qual a duração do curso? & Duração & tem duração \\
\hline Qual a carga horário do curso? & Carga Horária & tem carga horária \\
\hline Qual o nível do curso? & Nível & tem nível \\
\hline Qual o desempenho dos alunos? & Desempenho & tem desempenho \\
\hline O curso possui certificação? & Certificação & tem certificação \\
\hline
\end{tabular}

Fonte: Elaborado pelos autores.

\subsection{Hierarquia de classes}

As classes identificadas a partir dos termos da etapa anterior, foram hierarquizadas dando origem a taxonomia que suporta o desenvolvimento da ontologia do curso FCTEWeb. Para o desenvolvimento da taxonomia foi utilizado a ferramenta Protégé, para o aperfeiçoamento e manipulação das classes. Nesse processo, foram desconsideradas algumas classes que se julgou menos relevante para a representação da ontologia. A hierarquização procurou estabelecer sub-classes com forte relação com a classe principal. Todas as demais ligações entre classes foram estabelecidas por meio de relações, como mostra a Figura 1.

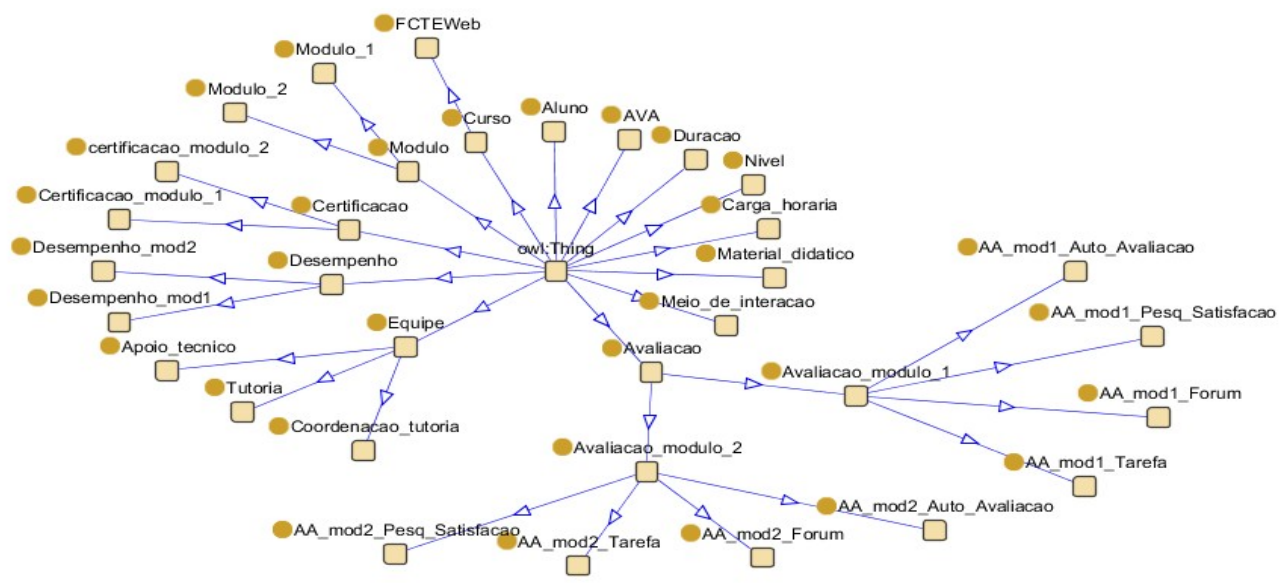

Figura 1 - Estrutura hierárquica da ontologia do curso FCTEWeb. Fonte: Elaborada pelos autores na ferramenta Protégé. 
Uma ontologia contém uma classe chamada owl:Thing. As classes OWL são interpretadas como conjuntos de indivíduos (ou conjunto de objetos). A classe owl:Thing é a classe que representa o conjunto que contém todos os indivíduos, uma vez que todas as classes são subclasses de owl:Thing (HORRIDGE, 2004).

Assim, para a ontologia do curso FCTEWeb, obtiveram-se treze classes principais (Aluno, AVA, Avaliação, Carga-horária, Certificação, Curso, Desempenho, Duração, Equipe, Material Didático, Meio de Interação, Módulo e Nível) e dezenove subclasses relacionadas às classes Avaliação, Certificação, Curso, Desempenho, Equipe e Módulo. Desta forma, obteve-se como resultado um total de trinta e duas subclasses da classe owl:Thing.

\subsection{Propriedades}

Propriedades são relações binárias que ligam uma instância de classe a outra instância de classe. Existem dois tipos principais de propriedades: propriedade de objetos, que conectam uma instância a outra; e propriedade de dados, que liga uma instância a um valor (HORRIDGE et al., 2004). Para fins deste estudo foram realizadas apenas propriedades de objetos.

Uma primeira identificação de relações foi feita logo após a extração dos vocábulos das perguntas de competência. Contudo, as perguntas de competência não permitem delimitar todas as relações possíveis entre as classes. As relações tornaram-se mais facilmente perceptíveis a partir da hierarquização das classes, sendo então formalizadas na ferramenta Protégé.

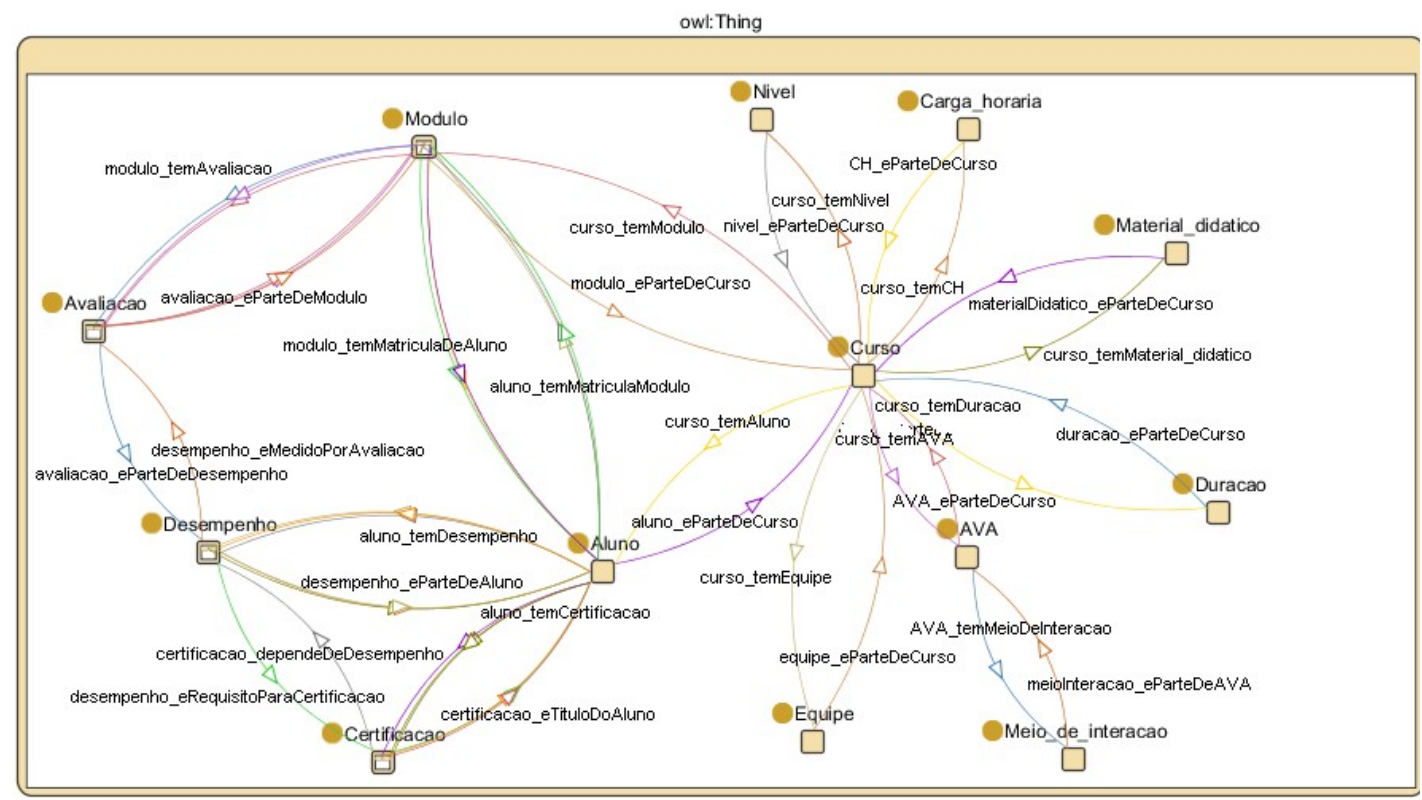

Figura 2 - Relação da classe Curso.

Fonte: Elaborada pelos autores na ferramenta Protégé.

A ontologia desenvolvida conta com um total de quarenta e seis relações, sendo estas diretas, isto é, que não possuem uma característica específica que as rege (HORRIDGE, 2004). As relações que ligam a classe Módulo à classe Avaliação, Aluno à classe Módulo, Aluno à classe Desempenho e Aluno à classe Certificação, possuem subrelações, que estão sinalizadas na Figura 2 como flecha dupla. 
Essas relações têm a finalidade de ligar uma instância (um indivíduo) de classe à outra instância de classe. Além disso, as relações possibilitam a criação de restrições, axiomas e regras lógicas.

\subsection{Instâncias}

As instâncias são os indivíduos pertencentes às classes. Isto é, as instâncias da classe Aluno, por exemplo, são os próprios alunos do curso FCTEWeb - módulo 1 e 2. A Figura 3 - ilustra as instâncias das classes Módulo e Curso.

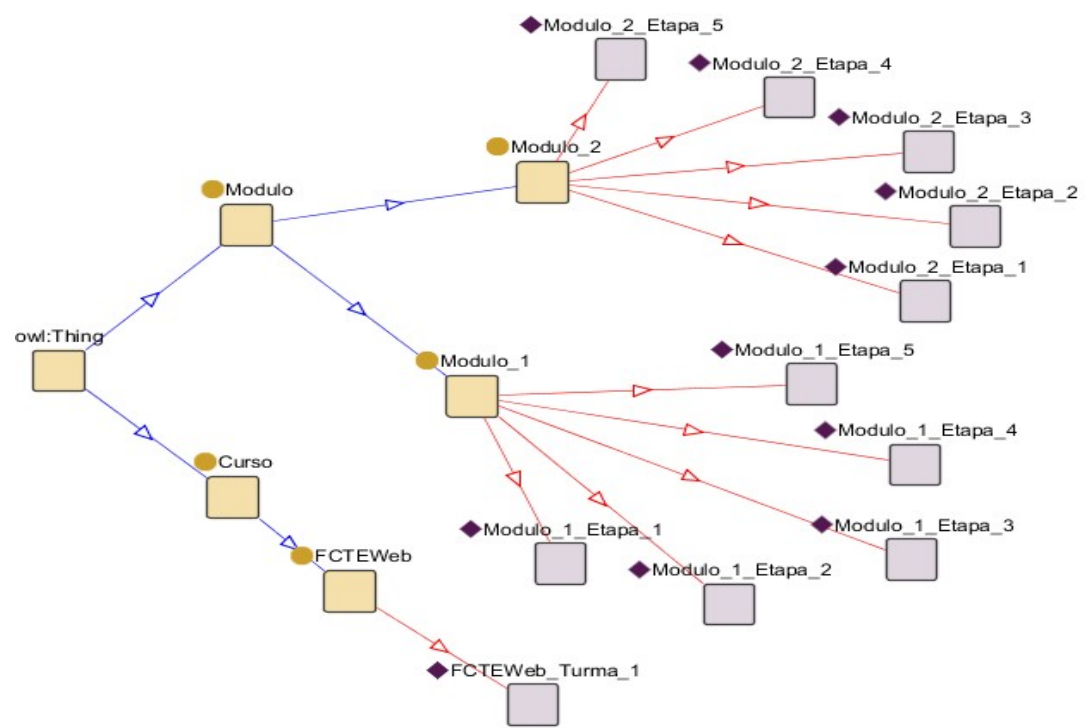

Figura 3 - Instâncias das classes Módulo e Curso.

Fonte: Elaborada pelos autores na ferramenta Protégé.

A ontologia desenvolvida, conta com um total de onze instâncias que ligam a classe Módulo à classe Curso de acordo com a definição do curso, ou seja, as instâncias fazem parte do conhecimento prévio existente ao se trabalhar a ontologia. Além disso, a instância aqui organizada para o curso FCTEWeb, permite uma análise em relação ao Módulo e Curso em suas diferentes instâncias.

\subsection{Discussão}

A ontologia desenvolvida representa a estrutura do curso FCTEWeb. Com base nessa estrutura, pode-se criar regras lógicas associadas à Ontologia, para automatizar algumas tarefas, por exemplo:

- Se Aluno tem desempenho no Módulo 1 maior ou igual a $60 \%$ então aluno tem matrícula no Módulo 2.

- Se Aluno tem desempenho no Módulo 1 maior ou igual a $60 \%$ então aluno tem certificação do Módulo 1.

- Se Aluno tem desempenho no Módulo 2 maior ou igual a 60\% então aluno tem certificação do Módulo 2.

Para a construção dessas regras tomam-se a classe Aluno e as relações

- “aluno_temDesempenhoModulo1”,“aluno_temDesempenhoModulo2”

- “aluno_temMatriculaModulo2”, "aluno_temCertificacaoModulo1”

- "aluno_temCertificacaoModulo2" 
Além disso, a ontologia pode apoiar na tomada de decisão dos gestores no que concerne a: (i) ajuste da grade curricular do curso; (ii) desenvolvimento de novos cursos; (iii) caracterização do perfil dos alunos; (iv) criação de um banco de vagas relacionados a atividades profissionais; entre outros.

\section{Conclusões}

O uso de ontologias para a representação do conhecimento apresenta grande potencial para auxiliar os coordenadores e gestores de cursos on-line e, também, oferece a possibilidade de se automatizar algumas atividades para automatizar algumas tarefas, como, a inscrição automática no módulo seguinte, se aprovado no anterior. Contudo, para que possa ser efetivo, é fundamental a participação e colaboração dos principais conhecedores do domínio do tema, desde os momentos iniciais do projeto. O uso de metodologias e ferramentas adequadas facilitam nas tarefas de aquisição e documentação do conhecimento explicitado como, relatórios do AVA. Também, podese produzir artefatos úteis para o compartilhamento e reutilização do conhecimento.

É importante ressaltar que, o mapeamento do conhecimento é uma atividade perene, que deve ser realizada periodicamente, atualizando, inclusive, sempre que necessário, os artefatos utilizados para a devida documentação. Desse modo, é importante a revisão periódica das ontologias, construídas pode permitir a otimização dos resultados obtidos com seu uso, bem como a construção e reconstrução do conhecimento. Esta análise permite, ainda, ir além e indicar que a ontologia ocupa posição de destaque numa proposta de gestão do conhecimento, haja vista que permite a organização de uma estrutura base para a construção, compartilhamento e disseminação das informações e do conhecimento contribuindo para a estruturação do conhecimento pré-existente.

\section{Referências}

ALMEIDA, M. B.; BAX, M. P. Uma visão geral sobre ontologias: pesquisa sobre definições, tipos, aplicações, métodos de avaliação e de construção. Revista Ciência da Informação, Brasília, v. 32, n. 3, p. 7-20, set./dez. 2003.

BERNERS-LEE, T.; HENDLER, J., LASSILA, O. The Semantic Web. Scientific American, p. 34-43, 2001.

BORST, W. N. Construction of Engineering Ontologies. 1997. Thesis (Ph. D.) University of Tweenty - Centre for Telematica and Information Technology, Enschede, Nederland, 1997.

CORCHO, O.; FERNÁDEZ-LÓPES, M.; GÓMEZ-PÉREZ, A. Methodologies, tools and languages for building ontologies. Where is their meeting point? IN: Data \&Kinowledge Engineering, n. 46, Elsevier Science, p. 41-64.

CUNHA, A. G. da. Dicionário etimológico da língua portuguesa. $4^{\mathrm{a}}$.ed. Rio de Janeiro: Lexikon, 2010.

FERNÁNDEZ-LÓPEZ, M. et al. Building a chemical ontology using methotology and the ontology design environment. [S. 1.]: IEEE Intelligent Systems \& their Applications, p. 37-46, jan./fev. 1999. 
FREITAS, F. L. G. Ontologias e a web semântica. In:Jornada de Mini-Cursos em Inteligência Artificial, SBC, v. 8, p. 1-52, 2003.

GOÑI, J. L.; FERNANDES, M. C. P.; LUCENA, C. J. P. Geração de Ontologias usando Protégé-2000 para Reuso de Conteúdos Educacionais numa Arquitetura Multiagente na Web Semântica. XIII Simpósio Brasileiro de Informática na Educação. Porto Alegre. 2002.

GRUBER, T. A translation approach to portable ontology specification.Knowledge Acquisition, Amsterdam, v. 5, n. 2, p. 199-220, 1993.

What is an ontology?1996. Disponível em: <http://www-

ksl.stanford.edu/kst/what-is-an-ontology.html>. Acesso em: 14 ago. 2013.

GUIZZARDI, G. Ontological Foundations for Structural Conceptual Models. Enschede: The Netherlands, 2005. Disponível em:

<http://doc.utwente.nl/50826/1/thesis_Guizzardi.pdf>. Acesso em: 08 set. 2013.

HORRIDGE, M. et al. A Practical Guide To Building OWL Ontologies using the Protege-OWL plugin and CO-ODE Tools. Edition 1.0. 2004.

MEDEIROS, M. F. Paradigma em educação à distância: processo reconstrutivista em ambientes colaborativos e interativos. In: Anais da III Jornadas de Educación a Distancia - Mercosur 99. Osormo, Chile, 1999.

MOLOSSI, S. Inserção da biblioteca digital de teses e dissertações no contexto da web semântica: construção e uso da ontologia. Dissertação de Mestrado no Programa de Pós-graduação em Ciência da Informação, UFSC, Florianópolis, SC, 2008.

MOORE, M.; KEARSLEY, G. Educação a distância: uma visão integrada. São Paulo: Thomson Learning, 2007.

NOY, F. N.; GUINNESS, D. L. Ontology development 101: a guide to create your first ontology. Disponível em: <http://ksl.stanford.edu/people/dlm/papers/ontologytutorial-noy-mcguinness. doc >. Acesso em: 4 set. 2013.

PEREIRA, A. K.; et al. A construção colaborativa do conhecimento através do ambiente virtual de aprendizagem. In: I Congresso Ibero-Americano de Gestão do Conhecimento e Inteligência Competitiva, 2006, Curitiba.

PROTÉGÉ. The Protégé ontology editor and knowledge acquisition system. 2001. Disponível em: http://protege.stanford.edu. Acesso em: 05 maio 2014.

RAUTENBERG, S., et al. Ferramenta OntoKEM: uma contribuição à Ciência da Informação para o desenvolvimento de ontologia. In: Perspectivas em Ciência da Informação, v. 15, n.1, p.239-258, 2010.

SARTORI, V. Comunidade virtual de prática como ferramenta de compartilhamento de conhecimento na educação a distância. Dissertação de Mestrado no Programa de Pós-graduação em Engenharia e Gestão do Conhecimento, Universidade Federal de Santa Catarina, Florianópolis, SC, 2012.

STUDER, R. et al. Knowledge Engineering: Principles and methods. Data \& Knowledge Engineering, v. 25, n. 1, p. 161-197, 1998. 